\title{
GESTIÓN DE LA CALIDAD BAJO LA NORMA ISO 9001 EN INSTITUCIONES PÚBLICAS DE EDUCACIÓN SUPERIOR DE MÉXICO
}

\section{Introducción}

El proceso de globalización que la humanidad ha experimentado en las últimas décadas se caracteriza por la integración de las economías locales en una economía de mercado mundial en donde las formas de producción y el flujo de capital evolucionan a una escala planetaria, desde una economía basada en la fabricación y la industria hacia otra basada en el conocimiento: la llamada «nueva economía» (Abreu, 2013).

Por otra parte, desde finales de los años noventa la globalización se ha convertido en un concepto relativo no solo en términos de la organización económica entre países, sino también en función de su organización política, social y cultural de las naciones. El orden jurídico y la normatividad también se ven afectados por el proceso de globalización, el que genera la necesidad de uniformizar y simplificar los procedimientos y las regulaciones, tanto regionales como nacionales e internacionales, hacia una mejora en la competitividad y la seguridad jurídica que conlleva, con tendencia hacia la universalización y el reconocimiento de los derechos fundamentales de los ciudadanos (Unesco, 2005).

Ahora bien, si vamos más atrás, a principios de los años ochenta, en el Reino Unido surge la Nueva Gestión Pública (NGP), la que en términos generales se refiere a las políticas gubernamentales que tienen como objetivo modernizar y hacer más eficaz el sector público, principalmente con un enfoque tendiente a solucionar problemas de tipo fiscal, con el fin de evitar la pérdida de dinamismo del crecimiento económico. Básicamente la teoría sostiene que la gestión del sector público orientada hacia el mercado dará lugar a una mayor rentabilidad para los gobiernos, sin tener efectos secundarios negativos en otros objetivos y consideraciones (Del Castillo, 2006; Hood, 1991). En comparación con otras teorías de la gestión pública, 
la NGP está encaminada hacia los resultados y la eficiencia por una mejor administración del recurso público y se dirige a los beneficiarios de los servicios públicos, como si estos fueran los clientes de productos y servicios y, a la inversa, se considera a los ciudadanos como si fueran accionistas. Este tipo de gestión se logra mediante la aplicación de la competitividad al interior de las organizaciones del sector público, como ocurre en el sector privado, pero haciendo hincapié en los principios de economía y de liderazgo (Gruening, 2001). Basados en lo anterior, los partidarios de la NGP afirman que las técnicas y prácticas del sector privado son directamente transferibles al sector público (Ferlie, Pettigrew, Ashburner, Fitzgerald, 1996).

En el contexto de la NGP Alan Scott (2013) observa que, de forma similar a otros proveedores de servicios públicos, las universidades debían ser expuestas simultáneamente tanto a la disciplina del mercado como también a sistemas más estrictos y transparentes de rendición de cuentas y auditoría; estos nuevos instrumentos de control se introducirían, paradójicamente, en nombre de la autonomía: las universidades debían ser liberadas del control directo del Estado, a cambio de aumentar la eficiencia y demostrar una mejora en las auditorías de la calidad docente y en los resultados de la investigación y su impacto.

En este sentido se reconoce desde hace tiempo que las instituciones educativas, tanto del sector público como las del privado, no son como las otras organizaciones, es decir, son más imprecisas que la mayoría, reflejan una gran cantidad de metas y objetivos contradictorios y, la mayoría de las veces, estos son indeterminados. Del mismo modo, se considera que las instituciones educativas son organizaciones con objetivos ambiguos, tecnologías inciertas, falta de coordinación de las actividades y se encuentran débilmente conectadas con los resultados esperados de la organización (Fusarelli y Johnson, 2004). Es por ello que, como en una anarquía organizada, existen conexiones sueltas entre la política, las acciones administrativas y académicas y la toma de decisiones que con frecuencia se aproxima a un cubo de basura, modelo en el que los problemas, las posibles soluciones y las oportunidades de elección interactúan para producir decisiones un tanto fortuitas (Cohen, March y Olsen, 1972). 
Es así que cuando se habla de un cambio en la educación superior, este se relaciona con la alteración de las reglas del juego, tanto en la organización académica como en el gobierno de las instituciones, donde debe existir congruencia entre el análisis de los mecanismos y los resultados esperados, partiendo de los procesos estratégicos hacia un cambio en la estructura formal, la cultura organizacional, las metas y la misión-visión para el logro de un cambio institucional que permita alcanzar el objetivo deseado: la calidad del servicio.

Ahora bien, como consecuencia del proceso de globalización, la relevancia adquirida por el concepto de calidad en la educación superior se ha basado en dar una respuesta adecuada a la necesidad de conocimiento que se atiende -ese producto valioso en la nueva economía-; sin embargo, debido a las múltiples facetas que presenta la naturaleza del concepto, no se ha llegado aún a un consenso y existen en la actualidad diversos enfoques, significados y aceptaciones (De la Orden, Asensio, Carballo, Fernández, Fuentes, García, Guardia y Navarro, 1997; Reimers y Villegas-Reimers, 2005; Senlle y Gutiérrez, 2005).

Por su parte, las instituciones de educación superior contemplan como una prioridad la gestión de la calidad, debido a que se les considera centros de capacitación de profesionales e investigadores de alto nivel por las organizaciones empresariales, la industria de la transformación y las dependencias gubernamentales, pero también representan unidades de producción de conocimiento y tecnología, aspectos esenciales para mantener el ritmo de desarrollo económico y elevar la calidad de vida del país. No obstante, para la comunidad en general el concepto de calidad aplicado a la educación superior se asocia directamente con el éxito que tengan los egresados en el mercado de trabajo, es decir, los interesados en recibir una educación superior con calidad esperan que el conocimiento y la formación recibidos estén en concordancia con las habilidades y competencias demandadas en el área laboral y de acuerdo con las necesidades regionales y globales (Reimers y Villegas Reimers, 2005; Senlle y Gutiérrez, 2005).

Debido a lo anterior, la calidad en la educación superior continúa siendo uno de los principales retos que enfrenta cualquier 
país, ya que las consecuencias de una educación deficiente en este rubro se relacionan con la lentitud o el bajo nivel de crecimiento cultural, social y económico, por lo que se considera que un país que no es capaz de desarrollar su propia tecnología, difícilmente podrá avanzar de manera competitiva en el ámbito de la nueva economía.

Por otra parte, los recursos que se invierten en la educación son de tal importancia que, necesariamente, se requiere evaluar y analizar su correcta aplicación e impacto en la sociedad, en donde la calidad de la educación tendrá efectos positivos. En la sociedad del conocimiento ya no se transige una economía cuya base sea el desperdicio, el retraso, los servicios deficientes, las falsas promesas o los presupuestos inadecuados o no sustentados en la realidad (Arcos, Hernández y Alcázar, 2009; Organización para la Cooperación y el Desarrollo Económicos [OCDE], 2012; Unesco, 2005).

En México, una república representativa y democrática conformada por un Distrito Federal y 31 estados libres unidos por un pacto federal, el Sistema Educativo Nacional (SEN), como en cualquier otro país, corresponde a un conjunto de normas, instituciones, recursos y tecnologías destinado a ofrecer servicios educativos a la población mexicana. El marco normativo se establece en los Artículos 3 y 31 de la Constitución Política de los Estados Unidos Mexicanos y se reglamenta en la Ley General de Educación, la que establece tres tipos de educación:

- Básica, conformada por los niveles de preescolar, primaria y secundaria.

- Media superior, que comprende el nivel de bachillerato y la educación profesional técnica.

- Superior, que incluye los niveles técnico superior o profesional asociado, licenciatura y posgrado.

Se ofrecen, además, los servicios de educación inicial, educación especial, educación para adultos y capacitación para el trabajo. Los servicios del Sistema Educativo Nacional son regulados por la Secretaría de Educación Pública (SEP) y son otorgados por la federación, los gobiernos estatales y municipales, por instituciones públicas, algunas autónomas y por privadas. Complementariamente dicha secretaría 
(2013) indica que la educación que se ofrece debe ser laica y se guía por el principio democrático, siendo este un sistema de vida basado en el mejoramiento económico, social y cultural de las personas.

Ahora bien, desde finales del siglo XX el sistema de educación superior en México ha presentado varios problemas que afectan la calidad de este nivel educativo, entre los que se pueden destacar la expansión no regulada del sistema, combinada con la construcción reactiva de una estructura burocrática, lo que ha generado desafíos a la calidad que, sumados a la insuficiencia de recursos, son causa de notorias deficiencias (Aréchiga y De Thierry, 2003; Ibarra, 2009). Como precedente de este fenómeno, el Programa Nacional de Educación 2001-2006 elaborado por la Secretaría de Educación Pública ofreció un diagnóstico de la problemática de cada nivel educativo que, en su momento, se convirtió en la guía que orientaba las acciones para superar los obstáculos.

En lo que se refiere a la educación superior, los problemas que aún persisten según Rubio (2006) se relacionan con los siguientes aspectos:

- la rigidez en los programas educativos,

- la baja eficiencia terminal,

- el desempleo y subempleo de los egresados,

- la falta de integración de las actividades de difusión con la docencia y la investigación,

- la ausencia de consolidación del servicio social,

- las deficiencias en la orientación vocacional,

- la carencia de integración de cuerpos académicos consolidados,

- la insuficiente producción de conocimiento,

- la debilidad en los programas de posgrado,

- la poca participación de la sociedad en el desarrollo de la educación superior y

- la consolidación insuficiente del sistema de evaluación y acreditación.

Del mismo modo, se reconoce que la estructura actual de las universidades en México no se encuentra en las condiciones deseadas 
para enfrentar los desafíos y competir por un posicionamiento en los nuevos mercados del conocimiento, como menciona Ibarra (2009). Sin embargo, es en las diversas formas de gobierno y administración de una institución de educación superior donde se encuentra su capacidad para conducir operativamente un proyecto institucional que oriente las acciones y las conductas que impactarán a la sociedad, generando de este modo el efecto deseado de solucionar problemas y satisfacer las necesidades y, consecuentemente, influyendo en el estilo de vida tanto de los individuos como de las comunidades.

Debido a lo anterior, una de las opciones que empieza a ser considerada por la mayoría como la forma más apropiada de gobierno de las instituciones de educación superior es aquella en la que se incluye la gestión de la calidad, partiendo desde la operación de procesos tales como el cambio organizacional (reingeniería y destrucción creativa, entre otros), el financiamiento, la relación con el entorno hasta la evaluación. Todas ellas son características del modelo que Clark (2000) denomina la «universidad emprendedora». Ibarra (2003) manifiesta al respecto que este modelo refuerza esa autonomía práctica que ubica a las instituciones de educación superior como ejecutoras de programas que responden esencialmente a demandas externas.

Ahora bien, sobre la base de los problemas detectados, la Secretaría de Educación Pública generó el Programa Integral de Fortalecimiento Institucional (PIFI), como una estrategia para apoyar a las instituciones de educación superior para que lograran mejores niveles de calidad en sus programas educativos y en los servicios que ofrecían. Mediante este programa las instituciones han recibido recursos adicionales en respuesta a las prioridades que derivan de un ejercicio de planeación estratégica participativa, en tanto que la cobertura y los beneficiarios de dicho programa incluyen a universidades públicas estatales, universidades públicas estatales de apoyo solidario, universidades politécnicas, universidades tecnológicas y otras instituciones afines (SEP, 2005).

A partir de su implementación en 2001, los procesos de evaluación y seguimiento del programa han sido un factor clave para la 
mejora de la calidad en las instituciones de educación superior del país. Sus métodos de evaluación y seguimiento se enfocan en el impacto del fortalecimiento institucional sobre el proceso de planeación estratégica participativa y el fortalecimiento de las dependencias de educación superior desarrolladas dentro del marco de dicho programa. Actualmente la iniciativa se encuentra en su décima etapa (SEP, 2012) y dentro de sus objetivos generales está consolidar los procesos de autoevaluación institucional, los de evaluación externa y los de mejora continua de la calidad, con el fin de:

- Conservar la acreditación de programas educativos que haya sido otorgada por organismos especializados reconocidos por el Consejo para la Acreditación de la Educación Superior (COPAES) y/o conservar la clasificación en el nivel 1 del Padrón de Programas Evaluados por los Comités Interinstitucionales para la Evaluación de la Educación Superior (CIEES).

- Mejorar y asegurar la calidad y permanencia de los programas educativos de posgrado que lograron su ingreso al Programa Nacional de Posgrados de Calidad (PNPC).

- Certificar, por la Norma Internacional ISO 9001: 2008 y los procesos académico-administrativos asociados.

- Consolidar la rendición de cuentas a la sociedad respecto de su funcionamiento.

Es por lo anterior que en las universidades las principales actividades realizadas en relación con la gestión de la calidad de la educación se agrupan en las siguientes categorías:

- La acreditación de programas educativos mediante el reconocimiento público de su calidad, con base en los indicadores establecidos por los organismos acreditadores que cuentan con el aval de las instancias gubernamentales.

- La certificación de procesos de administración y gestión, por medio de la implementación y certificación de sistemas de gestión de la calidad bajo la Norma ISO 9001 (Gobierno de los Estados Unidos Mexicanos [GEUM], 2007; SEP, 2005).

Debido al proceso de globalización y a la necesidad de uniformizar y simplificar los procedimientos y regulaciones, la 
serie de Normas ISO 9000 de la International Organization for Standardization (ISO) surge como respuesta a los requerimientos de los sistemas de gestión de la calidad en las organizaciones o empresas, además de ser una vía para reducir costos y mejorar los procesos de producción, tomando en cuenta que la calidad es un factor clave para la competitividad en cualquier mercado. En la actualidad el uso de esta norma representa un requisito fundamental para la aceptación de productos en muchas industrias, así como también está avalada por la Sociedad Americana de Calidad (ASQ), los Institutos Europeos de Estándares y el Comité Japonés de Estándares Industriales. Cabe mencionar que lo que usualmente se conoce como ISO 9000 no es una sola norma, sino un conjunto de ellas, una familia de estándares de aplicación y uso general cuyo objetivo principal es arraigar un sistema de gestión de la calidad dentro de una organización, incrementando con ello la productividad, reduciendo los costos innecesarios y asegurando la calidad de los procesos y productos, además de garantizar características como la protección al medioambiente, la seguridad, la fiabilidad, la eficiencia y la capacidad de intercambio a un costo económico conveniente.

Históricamente el desarrollo de las normas ha requerido de revisiones periódicas para decidir si es necesario modificarlas, mantenerlas, o bien, retirarlas. Como consecuencia de este mecanismo la Norma ISO 9001 ha pasado también por un proceso de revisión que finalmente concluyó con la publicación de la Norma ISO 9001: 2008, puesta en vigencia el 15 de noviembre de 2008 (Esponda, Palavicini y Navarrete, 2001; ISO, 2008).

Uno de los aportes significativos de la serie de Normas ISO 9000 que se verificó con el fin de conducir a las organizaciones hacia una mejora en el desempeño es el diseño de un sistema de gestión de la calidad basado en ocho principios de gestión que se indican a continuación:

1. Enfocarse hacia el cliente o usuario.

2. Liderazgo.

3. Involucrar al personal.

4. Enfoque basado en procesos. 
5. Administrar con enfoque de sistemas.

6. Mejorar continuamente.

7. Tomar decisiones basadas en hechos.

8. Beneficio mutuo en la relación con los proveedores.

Por otra parte, la principal característica de la Norma ISO 9001 se centra en la documentación, específicamente en el Manual de la gestión de la calidad en el que se menciona con claridad todo lo que es relevante para lograr la calidad de los productos o servicios que se ofrecen y garantizar con ello que exista un sistema de gestión de la calidad adecuado a la organización. Igualmente, en este documento se establecen los alcances, la política de calidad y los objetivos asociados a ella, así como también se describen los procesos que intervienen y sus interrelaciones (ISO, 2005). La elaboración de estos manuales no es fortuita, sino que por el contrario, exige una metodología y conocimientos y criterios organizacionales para documentar no solo lo que se desea hacer, sino también cómo se logrará, de modo de cerciorarse de que el personal involucrado cuente con el conocimiento de cómo hacerlo, de realizarlo tal como se planeó, de supervisar el trabajo realizado y verificar que lo hecho esté de acuerdo con lo que se dijo. En este sentido la persona que se dedique a normalizar debe ser conocedora de esta actividad, garantizando así la incorporación de mecanismos que se adapten a la realidad de los procesos, que sean útiles, de fácil entendimiento y no generen papeleo excesivo o una burocracia alterna (Esponda et al., 2001; ISO, 2005).

Es así que la búsqueda de la calidad en los bienes y servicios tiene su origen en procesos de tipo físico (tangible), donde el desarrollo tecnológico ha permitido obtener una confiabilidad, el control de los procesos de medición, en tanto que la forma de evaluar cuenta con una disciplina particular. Sin embargo, en relación con el ámbito de los servicios, se han detectado problemas por resolver, ya que en este aspecto es determinante la importancia del factor humano y, debido a la intangibilidad de este tipo de procesos, la dificultad de la medición es mayor, como es el caso de los procesos relacionados con las actividades académicas. Lo anterior ha provocado, por una parte, la necesidad de establecer una definición de los procesos que sea más precisa y, por otra, la naturaleza cambiante de muchos procesos 
ha ocasionado la necesidad de establecer diversos mecanismos para la mejora continua (ISO, 2005; Méndez, Jaramillo y Serrano, 2006). En cuanto a la clasificación de procesos se pueden distinguir los siguientes:

- Procesos estratégicos o de gestión, asociados al rumbo de la organización a mediano y largo plazo, misión-visión.

- Procesos clave, de realización o críticos, asociados a la operación, entrega de productos y servicios al cliente o al usuario. Los procesos clave o críticos se identifican porque agregan valor al cliente o usuario y regulan o crean insumos, los que a su vez permiten operar en procesos de valor agregado.

- Procesos de gestión de recursos, soporte o de apoyo a la operación, ya sea a los procesos clave o a los críticos, contribuyendo a su mejor funcionamiento (clientes internos).

- Procesos de medición, análisis y mejora, necesarios para recabar información acerca de los mismos, al mismo tiempo que medir, realizar el análisis del desempeño y la mejora de la eficacia y la eficiencia de la organización.

Deming (1986) considera que si no se puede describir lo que se está haciendo como un proceso, realmente no es posible saber lo que se está haciendo y para ello demostró que la producción es un sistema y no una secuencia de procesos mecánicos sin relación. Debido a lo anterior, el desempeño de una organización puede mejorarse por medio de la aplicación y el uso del enfoque basado en procesos, donde estos últimos se gestionan como un sistema gracias a la creación y entendimiento de una red de procesos y sus interacciones. Es por esto que la Norma ISO 9001 no se aplica a los procesos, ni a productos o a servicios, sino a los sistemas que los crean y administran, proporcionando la armonización de las normas de calidad en una escala internacional (ISO, 2005; ISO, 2008).

En una institución de educación superior los procesos clave son aquellos que se relacionan con atender la necesidad de conocimiento, impartirlo y su búsqueda mediante la investigación, por lo que constituye todo un reto para cualquier entidad educativa el cambio organizacional que significa implementar y dar seguimiento a un 
sistema de gestión de la calidad bajo la Norma ISO 9001. Pese a ello, es sin duda una plataforma fundamental para crear un sistema de gestión que se convierta en la directriz de una nueva práctica administrativa. No obstante, para el logro de los objetivos y metas establecidas en una institución de educación superior, no basta con una única calidad que debe desarrollar una institución (Esponda et al., 2001; ISO, 2005; ISO, 2008).

\section{Antecedentes}

Al interior de las instituciones de educación superior se llevan a cabo procesos de adaptación estratégicos a las nuevas realidades del siglo XXI, entre los que destaca la creación de sistemas de garantía de la calidad que aseguren ciertos niveles de excelencia en las instituciones. En los resultados de un estudio acerca de experiencias prácticas de instituciones de educación superior realizado en 30 países, Kasperaviciute (2013) observa que los principales motivos para la aplicación del modelo de la Fundación Europea para la Gestión de Calidad (EFQM) y/o el estándar de la Norma ISO 9001 se relacionan comúnmente con la competitividad en el mercado y las necesidades institucionales internas y de las partes interesadas, en tanto que identifica también que la problemática para la aplicación de estos medios se encuentra más frecuentemente relacionada con problemas institucionales que con cuestiones del modelo y/o el estándar.

Desde esta óptica, la implementación de cualquiera de estos medios en el contexto de estas instituciones condiciona los beneficios a determinados cambios organizacionales internos. Entre los beneficios más importantes que se pueden señalar están aquellos relacionados con los objetivos de los medios y los cambios culturales en la institución, ya que ellos conducen a la definición de un nuevo modelo de universidad, caracterizado por la globalización, la universalidad (esto es, sirviendo a todos y en todo momento) y la necesidad de dar respuesta a las nuevas demandas de la sociedad del conocimiento (FECIES, 2012; Mora, 2004).

Otra aportación significativa de la Norma ISO 9001 ha sido la aplicación de los conceptos Planificar-Hacer-Verificar-Actuar, 
metodología que Deming (1986) nos ofrece como el «círculo virtuoso de la mejora continua», cuyo objetivo es el logro de un aumento en la satisfacción del cliente o usuario, mediante el cumplimiento de sus requisitos. Sobre esta base, las instituciones educativas, atendiendo los lineamientos del Programa Integral de Fortalecimiento Institucional, definen periódicamente sus planes de desarrollo en los que se incluye la gestión de la calidad como una de sus políticas institucionales. Del mismo modo, desarrollan e implementan los sistemas que garantizan la calidad en sus unidades académicas y administrativas, utilizándolos como una herramienta para la búsqueda permanente de esos logros. Por último, como en todo proceso de mejora continua, la evaluación y el seguimiento proporcionan una forma de verificar y, en su momento, actuar (ISO, 2008).

Simultáneamente a los procesos de evaluación y seguimiento desarrollados dentro del marco del ya mencionado Programa Integral de Fortalecimiento Institucional, durante el segundo semestre del año 2007 se realizó una evaluación a los sistemas de garantía de la calidad implementados bajo la Norma ISO 9001: 2000 en la Universidad Autónoma de Baja California (UABC), mediante una investigación no experimental exploratoria verificada por una encuesta, con el diseño de un instrumento de medición autoadministrado y aplicado a los «representantes de la Dirección» de dichos sistemas.

Cabe mencionar que la UABC es una institución pública de educación superior fundada por un Congreso estatal, cuyo financiamiento está subsidiado por los gobiernos federal y estatal correspondientes; sus dependencias se encuentran distribuidas en tres campus que cubren los municipios de Ensenada, Mexicali, Playas de Rosarito, Tecate y Tijuana. Al momento del estudio, la institución estaba integrada por 38 unidades académicas (ocho escuelas, 22 facultades, siete institutos y el Centro de Investigaciones CulturalesMuseo) en tanto que su coordinación y administración es atendida por cinco unidades administrativas. Además de la Rectoría, existen tres Vicerrectorías distribuidas en Ensenada, Mexicali y Tijuana, en tanto que para los recursos financieros se cuenta con un patronato que los administra. Por último, según el Informe de Rectoría 2007, la matrícula total correspondiente al segundo semestre de 2007 fue de 
38.024 alumnos inscritos, atendidos por 4.234 académicos, de los cuales 1.043 son profesores de tiempo completo y reciben el apoyo de 1.557 trabajadores administrativos (UABC, 2007).

\section{Propósito de la investigación}

El propósito de la investigación fue el estudio de una muestra que permitiera conocer la situación de los 21 sistemas de gestión de la calidad implementados hasta ese momento en la UABC. El estudio contó con la respuesta de los representantes de la dirección de 18 sistemas, de los cuales ocho se encontraban implementados en unidades académicas y diez de ellos en unidades administrativas.

Ahora bien, ya que el representante de la dirección es la persona idónea para proporcionar información de un sistema de gestión de la calidad de conformidad con la Norma ISO 9001: 2008, es a quien se le confiere, con independencia de otras obligaciones y funciones, la responsabilidad y autoridad para asegurarse de:

- El establecimiento, implementación y mantención de los procesos necesarios para el sistema.

- Informar a los directivos respecto del desempeño del sistema de gestión de la calidad y de cualquier necesidad de mejora.

- Promover la toma de conciencia de los requisitos del cliente y/o usuario en todos los niveles de la organización (ISO, 2008).

Para contrastar las hipótesis, se consideraron las actividades encaminadas hacia el desarrollo de una cultura de la calidad en la institución, así como también los procesos, su implementación, sus interrelaciones y las formas de comunicación con el cliente o usuario. La relevancia de este estudio acerca de los sistemas de gestión de la calidad implementados en una institución pública de educación superior radica en la diversidad que existe, tanto en la definición del concepto de calidad, como en los métodos para lograrla, así como en las diversas formas de darle seguimiento y detectar oportunidades de mejora, proporcionado así elementos para adecuar o reorientar estas acciones y lograr los objetivos establecidos, completando el «círculo virtuoso de la mejora continua». 
En los resultados de la estadística descriptiva del estudio se detectó que del total de la muestra, 17 sistemas estaban certificados y uno en proceso de serlo. Por otra parte, el hallazgo relevante fue la ausencia de procesos relacionados con las actividades académicas.

Los datos obtenidos de la investigación aportaron evidencia cuantitativa y cualitativa para contrastar las hipótesis de trabajo y, como resultado de ello, se aceptaron las hipótesis nulas:

- En la UABC no se promueve el desarrollo de una cultura de la calidad.

- En los sistemas de gestión de la calidad implementados en la UABC no se aplica un enfoque basado en procesos.

- Los requisitos de los productos o servicios que se ofrecen en la UABC no se determinan estableciendo una comunicación con el cliente o usuario.

Por último, siendo inesperados los resultados del estudio se sugiere la continuación de trabajos de investigación relacionados con la gestión de la calidad en las instituciones públicas de educación superior en el contexto nacional, de modo que muestren evidencia del desempeño de los sistemas de gestión de la calidad implementados en ellas bajo la Norma ISO 9001: 2008.

\section{Planteamiento del problema}

En la actualidad se considera que las universidades mejor clasificadas son aquellas que contribuyen significativamente al avance del conocimiento mediante la investigación, porque enseñan con los planes de estudio y los métodos pedagógicos más innovadores bajo las condiciones más favorables, haciendo de la investigación una parte integrante de la enseñanza, por lo que forman egresados que sobresalen exitosamente en escenarios altamente competitivos (Salmi, 2009; Senlle y Gutiérrez, 2005).

Sobre la base de lo anterior, la necesidad de calidad en la educación superior adquiere singular relevancia, ya que se espera asegurar la competitividad de la economía nacional gracias al aprovechamiento del recurso humano. Además, la calidad y la 
excelencia se visualizan como las metas deseables en toda institución educativa, como ocurre en cualquier organización o empresa. Sin embargo el problema radica en que, al tratar de determinar las acciones de mejora para lograr las metas, no se logra un consenso en la definición de lo que significa la calidad en la educación y, en consecuencia, se difiere al determinar cuáles son los métodos idóneos para obtener una educación de calidad y en cómo evaluar este proceso para el logro de la mejora continua (De la Orden et al., 1997; Deming, 1986; Reimers y Villegas Reimers, 2005; Senlle y Gutiérrez, 2005).

Es necesario, pues, que en primera instancia las instituciones de educación superior logren un consenso para definir lo que significa calidad en la educación y el aprendizaje, de modo de proporcionar el conocimiento dirigido al desarrollo del recurso humano y de ese modo brindar diversas oportunidades de aprendizaje que efectivamente expandan las opciones y la libertad de las personas. Dicha definición debiera estar estrechamente relacionada con el propósito de la educación, los valores, los criterios normativos y demostrar una congruencia entre el conocimiento que se imparte y las habilidades y competencias demandadas en el área laboral por parte de la sociedad (De la Orden et al., 1997). La calidad y excelencia que distingue a las instituciones de educación superior, sin ser cuestionadas su eficiencia y eficacia, es aquella que tiene relación con estos propósitos, con la planificación estratégica (de políticas y prácticas pedagógicas) que contribuye al logro de dichos propósitos y que es compartida por el personal en los distintos niveles de actuación (Crosby, 1987; Deming 1986; Reimers y Villegas Reimers, 2005).

La decisión de implementar sistemas de gestión de la calidad bajo la Norma ISO 9000 para la mejora de la calidad en la educación superior debe ser, entonces, una decisión estratégica de la institución. Asimismo, la generación de sistemas de gestión de la calidad debe ser en su momento una experiencia única e irrepetible para cada organización, ya que se sabe que tratar de implementar un sistema que no haya sido el generado por la misma institución, ya sea copiado o adaptado, solo lleva al fracaso (Cisneros, 2003; Esponda et al., 2001). 
De este modo, la presente investigación comprende una evaluación a los sistemas de gestión de la calidad implementados en las instituciones públicas de educación superior del país y tiene por objetivo conocer si la implementación y certificación de dichos sistemas bajo la Norma ISO 9001: 2008 se utiliza como una herramienta valiosa para el logro de la mejora de la calidad en la educación superior, o bien, si solo se utiliza para cumplir con el requisito de una política pública. Por ello, se plantean las siguientes hipótesis:

- Hipótesis 1: En las instituciones públicas de educación superior se promueve el desarrollo de una cultura de la calidad mediante un liderazgo comprometido con la gestión de la misma (Crosby, 1987; Deming, 1986; Ishikawa, 1988; Juran y Gryna, 1993).

- Hipótesis 2: En las instituciones públicas de educación superior se mantiene un sistema de gestión de la calidad eficaz, con un enfoque basado en procesos que considera la comprensión y el cumplimiento de los requisitos establecidos por el cliente o usuario y la necesidad de atender a los procesos que aporten valor al sistema (Deming, 1986; ISO, 2008; Juran y Gryna, 1993; Méndez et al., 2006).

- Hipótesis 3: En las instituciones públicas de educación superior se aplica un enfoque orientado al cliente o usuario, conociendo sus requisitos y comprendiendo sus necesidades con el fin de satisfacerlos (Deming, 1986; Ishikawa, 1988; ISO, 2008).

A partir de los resultados del análisis de la información que generó el estudio, se obtuvieron los elementos necesarios para contar con un panorama general respecto del desempeño de los sistemas de gestión de la calidad implementados en las instituciones públicas de educación superior. En este sentido se pueden plantear recomendaciones y acciones a seguir, así como también ofrecer algunas observaciones que pudieran generar oportunidades de mejora a los sistemas y así adecuar o reorientar las iniciativas dirigidas hacia el logro de la calidad en estas organizaciones, completando de este modo el «círculo virtuoso de la mejora continua» (Deming, 1986).

Del mismo modo, el estudio aportó una forma de evaluación a los sistemas de gestión de la calidad sin representar una auditoría de calidad de tercera parte (extrínseca), obteniendo valiosa información 
para la generación de oportunidades de mejora y retroalimentación a los propios sistemas (ISO, 2008).

\section{Método}

El estudio correspondió a una investigación no experimental cuantitativa descriptiva por medio de la encuesta, para lo que diseñó el instrumento de medición aplicado en 2007 a los sistemas de gestión de la calidad de la UABC. Para contrastar las hipótesis se utilizó la prueba de la Ji-cuadrada de Pearson con tablas de contingencia (Hernández, Fernández, Baptista, 2006), analizándose las variables relacionadas con los siguientes aspectos:

- Las actividades que se realizaron para el desarrollo de una cultura de la calidad.

- La implementación de una estructura administrativa para la gestión de la calidad.

- La definición de los procesos del sistema de gestión de la calidad.

- El proceso de implementación y los indicadores del sistema.

- Las actividades que se realizan para establecer la comunicación con el cliente y/o usuario.

- La formación y/o capacitación para la gestión de las relaciones con el cliente y/o usuario.

\section{Sujetos}

Siendo la unidad de análisis los sistemas de gestión de la calidad implementados en las instituciones públicas de educación superior, como ya se mencionó la persona idónea para proporcionar la información relativa al tema es el coordinador o el representante de la dirección del sistema. Ahora bien, en conformidad con la Norma ISO 9001:2008 la responsabilidad de dicho representante puede incluir relaciones con partes externas acerca de asuntos relacionados con el sistema de gestión de la calidad.

Con el fin de extraer una muestra de estos sistemas implementados en las universidades públicas del contexto nacional, la información obtenida del portal de la Subsecretaría de Educación 
Superior perteneciente a la Secretaría de Educación Pública arrojó que el país cuenta con 43 universidades públicas estatales que desarrollan las funciones de docencia y la generación y aplicación innovadora del conocimiento, así como también iniciativas de extensión y difusión de la cultura, lo que llevó a incluir en el estudio a dos universidades públicas del Distrito Federal.

\section{Materiales}

El instrumento de medición aplicado a los sistemas de gestión de la calidad de la UABC corresponde a un cuestionario de preguntas cerradas y mixtas, conformado por el encabezado, donde se menciona el objetivo de la encuesta y las preguntas demográficas o de ubicación del sujeto encuestado, los datos generales de la unidad académica o administrativa a la que pertenece el sistema y la fecha de aplicación, entre otros aspectos; y el cuerpo, integrado por 27 reactivos, divididos en cinco secciones:

- Información general. Considera seis reactivos relacionados con datos generales acerca de los sistemas en investigación, como el tipo de unidad (académica o administrativa) y el número del personal adscrito, el número del personal que interviene en el sistema, el universo de atención del sistema y las fechas de implementación y de certificación, entre otros.

- Sobre calidad. Contempla cinco reactivos relacionados con la implementación de la infraestructura física y administrativa para la gestión de la calidad, el tipo y la frecuencia de actividades que se realizan para el desarrollo de una cultura de la calidad en la institución, el número del personal involucrado en estas actividades y el tipo y la frecuencia de capacitación o formación proporcionada al personal.

- El sistema. Este involucra seis reactivos relacionados con los procesos del sistema de gestión de la calidad, el número y tipo de procesos y sus interrelaciones, los correspondientes indicadores de calidad del sistema, los datos acerca de la planificación para ampliar sus alcances y la capacitación o formación oportuna del personal involucrado en estas actividades.

- Comunicación con el cliente/usuario. Incluye tres reactivos 
relacionados con el tipo y la frecuencia de aplicación de actividades para establecer la comunicación y determinar las necesidades del cliente o usuario, así como también la capacitación o formación oportuna del personal involucrado en estas actividades.

- Tu opinión es importante. Contempla reactivos con preguntas acerca de la opinión o las actitudes relacionadas con la experiencia de ser un representante de la dirección y el desarrollo de una cultura de la calidad en la institución. El cuestionario pasó por un proceso de revisión, adaptándose su redacción para que fuera aplicado a diversas instituciones públicas de educación superior. Con el fin de obtener los datos de forma objetiva, se aplicó el cuestionario en la UABC de forma similar a la de la investigación exploratoria, bajo la forma de un sondeo autoadministrado entregado vía electrónica. En el encabezado del cuestionario se indicó el objetivo de la investigación, así como la instrucción para que los casilleros fueran llenados por cada sistema de gestión de la calidad implementado bajo la Norma ISO 9001 por parte del correspondiente coordinador o representante de la dirección.

\section{Procedimientos}

En primera instancia se estableció un contacto con las instituciones públicas de educación superior consideradas para el estudio, a partir de los enlaces que se indicaban en el portal de la Secretaría de Educación Pública (2012). La búsqueda se realizó por medio de un buscador de internet que localizó la página de transparencia de cada institución, el correo electrónico y la vía telefónica, entre otros datos de interés. La información se recuperó durante el período correspondiente a los meses de febrero a octubre del 2012, al cabo del cual se logró reunir las respuestas de 22 universidades públicas que proporcionaron datos de 25 sistemas de gestión de la calidad implementados. Una vez realizada la captura de datos, se detectó que el cuestionario fue respondido en su totalidad en el 88\% de los casos, según se observa en el resumen del procesamiento de los casos representado en la Figura 1. 
Figura 1: Resultados tras la aplicación del instrumento de confiabilidad Análisis de confiabilidad Advertencia

Todas las siguientes variables constitutivas tienen una varianza cero y se eliminarán de la escala: El sistema de gestión de la calidad implementado ¿actualmente se encuentra certificado?

Escala: TODAS LAS VARIABLES

Resumen del procesamiento de los casos

\begin{tabular}{|c|c|c|c|}
\hline \multicolumn{2}{|c|}{} & $\mathrm{N}^{\circ}$ & $\%$ \\
\hline Casos & Válidos & 22 & 88.0 \\
\hline & Excluidos* & 3 & 12.0 \\
\hline & Total & 25 & 100.0 \\
\hline \multicolumn{2}{|c|}{ * Eliminación por lista basada en todas las variables del procedimiento. } \\
\hline
\end{tabular}

Alfa:

\begin{tabular}{|l|c|c||c|c|c|c|}
\hline \multicolumn{3}{|c||}{ Estadísticos de fiabilidad } & \multicolumn{3}{|c|}{ Estadísticos de la escala } \\
\hline $\begin{array}{l}\text { Alfa de } \\
\text { Cronbach }\end{array}$ & $\begin{array}{l}\text { Alfa de } \\
\text { Cronbach } \\
\text { basada en los } \\
\text { elementos } \\
\text { tipificados }\end{array}$ & $\begin{array}{l}N^{\circ} \text { de } \\
\text { elementos }\end{array}$ & Media & Varianza & Desviación típica & $N^{\circ}$ de elementos \\
\hline .835 & .854 & 35 & 84.55 & 279.307 & 16.712 & 35 \\
\hline
\end{tabular}

Fuente: Elaboración propia.

La validez y confiabilidad del instrumento de medición se obtuvo mediante el coeficiente Alfa de Cronbach; en tanto que los coeficientes de correlación variaron entre 0 (nula confiabilidad) y 1 (confiabilidad total). No existe una regla que indique a partir de qué valor existe o no confiabilidad, pero por lo general se considera que si se obtiene 0,25 en el coeficiente de correlación la confiabilidad es baja; si se obtiene un 0,50 sería media o regular; si es mayor a 0,75 tendría una confiabilidad aceptable; y si es de 0,90 la confiabilidad es elevada. Por otra parte la confiabilidad varía también de acuerdo con el número de variables o ítems que se incluya en el instrumento de medición, así entre mayor sea el número de dichas variables, mayor será la confiabilidad.

Finalmente, al ejecutar el análisis de fiabilidad con el programa SPSS, considerando las 36 variables en escala ordinal, se obtuvo el coeficiente Alfa de Cronbach con un valor de 0,835, con lo que se puede afirmar que el instrumento de medición era confiable, como se indica en la Figura 1 (Hernández et al., 2006; Pérez, 1999). 
Para que se verificaran las relaciones entre las variables consideradas para el estudio se aplicaron pruebas no paramétricas, utilizando la prueba de la Ji-cuadrada de Pearson con tablas de contingencia, las que no requieren de presupuestos acerca de la forma de distribución poblacional, aceptan distribuciones no normales, pueden analizar datos ordinales o nominales y las variables no necesariamente tienen que estar medidas en un nivel por intervalos de confianza o razón.

La Ji-cuadrada de Pearson prueba de independencia de datos categóricos, partiendo del supuesto de la no relación entre variables y luego analizando si las frecuencias observadas son diferentes de lo que pudiera esperarse en caso de correlación. Ahora bien, para analizar la relación de dependencia o independencia entre dos variables cualitativas nominales o factores es necesario estudiar su distribución conjunta o tabla de contingencia. En este sentido, al correr el programa SPSS este arroja el valor de Ji-cuadrada calculada, por lo que para saber si un valor de Ji-cuadrada es o no significativo se calculan los grados de libertad, aplicando la fórmula $g l=(r-1)(c-$ 1), donde «r» es el número de renglones de la tabla de contingencia $\mathrm{y}$ «c» el número de columnas. Al establecer el nivel de significancia $\alpha$ sumado al dato de los grados de libertad, se busca Ji-cuadrada $(\alpha$, gl) en las Tablas de valores críticos de la distribución Ji-cuadrada: si el valor calculado de Ji-cuadrada es igual o superior al valor encontrado en dichas tablas, se dice que las variables están relacionadas, con una Ji-cuadrada significativa y se acepta la hipótesis de investigación (Hernández et al., 2006; Pérez, 1999).

\section{Resultados}

En los resultados de la estadística descriptiva se detectó que los sistemas de gestión de la calidad se encuentran implementados en todas las unidades administrativas de la muestra y cuentan con su certificación vigente. Dentro de los hallazgos relevantes se observa que solo en dos casos se incluyen procesos relativos a la docencia y a la investigación. También se observa que en el 92\% de los casos se proporciona el dato concerniente al número de procesos clave del sistema de gestión de la calidad, aunque el porcentaje disminuye a $68 \%$ 
en cuanto a proporcionar la relación de procesos del sistema. Pese a que en 17 de los casos se manifiesta que sí se proporciona la relación de procesos, solo en 14 de ellos esta incluyó información adicional sobre los mismos, sus objetivos, la definición del cliente/usuario del proceso o los resultados esperados y los obtenidos.

Se considera que el contraste de las hipótesis constituye la acción para establecer la veracidad de los enunciados singulares del tipo, las que permiten utilizar la experiencia como la única base para distinguir lo verdadero de lo falso. Tradicionalmente el contraste se efectuaba mediante la aplicación de diversas pruebas lógicas bajo los diversos métodos: la observación, el experimento, la documentación y el muestreo. Sin embargo en la actualidad, con el avance de la estocástica en el campo de la estadística en donde las pruebas para contrastar las hipótesis no solo abarcan los aspectos cuantitativos, sino también los cualitativos, sus variables, indicadores e ítems, es posible determinar las medidas que prueban la validez o invalidez de las hipótesis de diversas formas, para lo que se utiliza la aplicación de la prueba de la Ji-cuadrada de Pearson con tablas de contingencia (Hernández et al., 2006).

\section{Sobre el desarrollo de una cultura de la calidad}

Planteamiento de hipótesis:

Hipótesis 1: En las instituciones públicas de educación superior se promueve el desarrollo de una cultura de la calidad con la implementación de sistemas de gestión de la calidad mediante un liderazgo comprometido.

Hipótesis 10: En las instituciones públicas de educación superior no se promueve el desarrollo de una cultura de la calidad con la implementación de sistemas de gestión de la calidad mediante un liderazgo comprometido.

Se eligieron las variables relativas a las actividades para el desarrollo de una cultura de la calidad, agrupándolas en la primera variable cualitativa nominal; del mismo modo, se eligieron las variables respecto de la implementación de la estructura administrativa para la gestión de la calidad para la segunda variable cualitativa nominal, 
de donde se obtuvo la Ji-cuadrada calculada igual a 35,625, con 28 grados de libertad. Para calcular la zona de rechazo se estableció un nivel de significancia alfa igual a 0,05, lo que dio un valor para Jicuadrada $(0,05,28)$ en las Tablas de valores críticos de la distribución Ji-cuadrada igual a 41,337. Debido a que Ji-cuadrada calculada con un valor de 35,625 es menor a la Ji-cuadrada $(0,05,28)$ con un valor de 41,337, se acepta la hipótesis nula «En las instituciones públicas de educación superior no se promueve el desarrollo de una cultura de la calidad con la implementación de sistemas de gestión de la calidad mediante un liderazgo comprometido». Ver Tabla 1.

Tabla 1: Sobre el desarrollo de una cultura de la calidad. Tabla de contingencia

\begin{tabular}{|c|c|c|c|c|c|c|c|c|c|c|c|c|c|c|c|c|c|c|}
\hline \multicolumn{19}{|c|}{ Sobre el desarrollo de una cultura de la calidad } \\
\hline & & & \multirow[b]{2}{*}{2} & \multirow[b]{2}{*}{3} & \multicolumn{13}{|c|}{ Actividades para el desarrollo de una cultura de la calidad } & \multirow{2}{*}{ Total } \\
\hline & & & & & 3 & 3 & 4 & 4 & 4 & 4 & 4 & 5 & 5 & 5 & 5 & 5 & 6 & \\
\hline \multirow{6}{*}{ 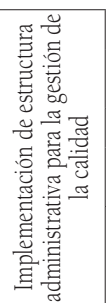 } & \multirow[b]{2}{*}{1} & Recuento & 1 & 1 & 1 & 4 & 2 & 1 & 0 & 1 & 1 & 0 & 1 & 0 & 3 & 0 & 0 & 16 \\
\hline & & $\begin{array}{l}\begin{array}{l}\text { Frecuencia } \\
\text { esperada }\end{array} \\
\end{array}$ & 6 & 6 & .6 & 2.6 & 1.3 & 1.3 & .6 & 1.3 & 1.3 & .6 & 1.3 & .6 & 1.9 & .6 & .6 & 16.0 \\
\hline & \multirow[b]{2}{*}{2} & Recuento & 0 & 0 & 0 & 0 & 0 & 1 & 1 & 1 & 0 & 1 & 0 & 0 & 0 & 0 & 0 & 4 \\
\hline & & $\begin{array}{l}\text { Frecuencia } \\
\text { esperada }\end{array}$ & 2 & 2 & .2 & .6 & .3 & .3 & 2 & .3 & .2 & .3 & .3 & .2 & .5 & .2 & .2 & 4.0 \\
\hline & \multirow[b]{2}{*}{3} & Recuento & 0 & 0 & 0 & 0 & 0 & 0 & 0 & 0 & 0 & 1 & 1 & 1 & 0 & 1 & 1 & 5 \\
\hline & & $\begin{array}{l}\text { Frecuencia } \\
\text { esperada }\end{array}$ & 2 & 2 & .2 & .8 & .4 & .4 & .2 & .4 & .2 & .4 & .4 & .2 & .6 & .2 & .2 & 5.0 \\
\hline \multirow{2}{*}{ Total } & & Recuento & 1 & 1 & 1 & 4 & 2 & 2 & 1 & 2 & 1 & 2 & 2 & 1 & 3 & 1 & 1 & 25 \\
\hline & & $\begin{array}{l}\text { Frecuencia } \\
\text { esperada }\end{array}$ & 0 & 0 & 1.0 & 4.0 & 2.0 & 2.0 & 1.0 & 2.0 & 1.0 & 1.0 & 2.0 & 1.0 & 3.0 & 1.0 & 1.0 & 25.0 \\
\hline
\end{tabular}

\begin{tabular}{|l|c|c|c|}
\hline \multicolumn{4}{|c|}{ Pruebas de Ji-cuadrada } \\
\hline \multicolumn{1}{|c|}{ I. } & Valor & $\mathrm{gl}$ & Sig. asintótica (bilateral) \\
\hline Ji-cuadrada de Pearson & $35.625^{*}$ & 28 & .152 \\
\hline Razón de verosimilitudes & 33.946 & 28 & .203 \\
\hline Asociación lineal por lineal & 7.026 & 1 & .008 \\
\hline $\mathrm{N}^{\circ}$ de casos válidos & 25 & \\
\hline$* 45$ casillas (100.0\%) tienen una frecuencia esperada inferior a 5. La frecuencia mínima esperada es .16. \\
\hline
\end{tabular}

Fuente: Elaboración propia.

\section{El sistema, aplicación de un enfoque basado en procesos}

Planteamiento de hipótesis:

Hipótesis 2: En los sistemas de gestión de la calidad implementados en las instituciones públicas de 
educación superior se aplica un enfoque basado en procesos.

Hipótesis 20: En los sistemas de gestión de la calidad implementados en las instituciones públicas de educación superior no se aplica un enfoque basado en procesos.

En este caso se eligieron las variables relativas a la definición de procesos del sistema de gestión de la calidad para agruparlas en la primera variable cualitativa nominal; de la misma forma, se escogieron las variables acerca del proceso de implementación e indicadores del sistema de gestión de la calidad para la segunda variable cualitativa nominal. Al correr el programa SPSS se obtuvo una Ji-cuadrada calculada igual a 14,413 con 16 grados de libertad; en tanto que al calcular la zona de rechazo se aplicó nuevamente un nivel de significancia alfa igual a 0,05 , lo que dio un valor para Ji-cuadrada $(0,05,16)$ en las tablas igual a 26,296 . Se observa que Ji-cuadrada calculada con un valor de 14,413 es menor a Ji-cuadrada $(0,05,16)$ en las tablas con un valor de 26,296, por lo tanto, se acepta la hipótesis nula «En los sistemas de gestión de la calidad implementados en las instituciones públicas de educación superior no se aplica un enfoque basado en procesos». Ver Tabla 2. 
Tabla 2: El sistema, aplicación de un enfoque basado en procesos. Tabla de contingencia

\begin{tabular}{|c|c|c|c|c|c|c|c|c|}
\hline \multicolumn{9}{|c|}{ El sistema, aplicación de un enfoque basado en procesos } \\
\hline \multirow{2}{*}{\multicolumn{3}{|c|}{ II }} & \multicolumn{5}{|c|}{ Definición de procesos del SGC } & \multirow{3}{*}{$\frac{\text { Total }}{8}$} \\
\hline & & & 1 & 2 & 2 & 3 & 3 & \\
\hline \multirow{10}{*}{ 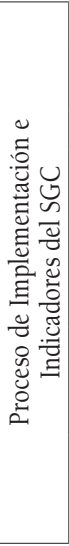 } & & Recuento & 6 & 2 & 0 & 0 & 0 & \\
\hline & 1 & $\begin{array}{l}\text { Frecuencia } \\
\text { esperada }\end{array}$ & 4.5 & 1.9 & 1.0 & .3 & .3 & 8.0 \\
\hline & & Recuento & 3 & 3 & 1 & 0 & 0 & 7 \\
\hline & 2 & $\begin{array}{l}\text { Frecuencia } \\
\text { esperada }\end{array}$ & 3.9 & 1.7 & .8 & .3 & .3 & 7.0 \\
\hline & & Recuento & 4 & 0 & 1 & 1 & 1 & 7 \\
\hline & 2 & $\begin{array}{l}\text { Frecuencia } \\
\text { esperada }\end{array}$ & 3.9 & 1.7 & .8 & .3 & .3 & 7.0 \\
\hline & & Recuento & 0 & 1 & 1 & 0 & 0 & 2 \\
\hline & 3 & $\begin{array}{l}\text { Frecuencia } \\
\text { esperada }\end{array}$ & 1.1 & .5 & .2 & .1 & .1 & 2.0 \\
\hline & & Recuento & 1 & 0 & 0 & 0 & 0 & 1 \\
\hline & 3 & $\begin{array}{l}\text { Frecuencia } \\
\text { esperada }\end{array}$ & .6 & .2 & .1 & .0 & .0 & 1.0 \\
\hline \multirow{2}{*}{\multicolumn{2}{|c|}{ Total }} & Recuento & 14 & 6 & 3 & 1 & 1 & 25 \\
\hline & & $\begin{array}{l}\text { Frecuencia } \\
\text { esperada }\end{array}$ & 14.0 & 6.0 & 3.0 & 1.0 & 1.0 & 25.0 \\
\hline
\end{tabular}

\begin{tabular}{|l|c|c|c|}
\hline \multicolumn{4}{|c|}{ Pruebas de Ji-cuadrada } \\
\hline & Valor & gl & Sig. asintótica (bilateral) \\
\hline Ji-cuadrada de Pearson & $14.413^{*}$ & 16 & .568 \\
\hline Razón de verosimilitudes & 16.976 & 16 & .387 \\
\hline Asociación lineal por lineal & 1.959 & 1 & .162 \\
\hline $\mathrm{N}^{o}$ de casos válidos & 25 & & \\
\hline * 25 casillas (100.0\%) tienen una frecuencia esperada inferior a 5. La frecuencia mínima esperada es .04.
\end{tabular}

Fuente: Elaboración propia.

\section{Comunicación con el cliente o usuario}

Planteamiento de hipótesis:

Hipótesis 3: Los requisitos de los productos o servicios que se ofrecen en las instituciones públicas de educación superior se determinan aplicando un enfoque al clienteusuario.

Hipótesis 30: Los requisitos de los productos o servicios que se ofrecen en las instituciones públicas de educación superior no se determinan aplicando un enfoque al cliente-usuario. 
En este caso se eligieron las variables relacionadas con las actividades para establecer la comunicación con el cliente o usuario, agrupándolas en la primera variable cualitativa nominal; de la misma forma se escogieron las variables relativas a la formación/ capacitación para la gestión de las relaciones con el cliente o usuario, para agruparlas en la segunda variable cualitativa nominal. Se obtuvo Ji-cuadrada calculada igual a 97,309 con 65 grados de libertad. Al calcular la zona de rechazo con un nivel de significancia alfa igual a 0,05 , dio un valor para Ji-cuadrada $(0,05,65)$ en las tablas igual a 84,821. Debido a que Ji-cuadrada calculada con un valor de 97,309 es mayor a Ji-cuadrada $(0,05,65)$ en las tablas con un valor de 84,821 , se acepta la hipótesis de investigación «Los requisitos de los productos o servicios que se ofrecen en las instituciones públicas de educación superior se determinan aplicando un enfoque al cliente-usuario». Ver Tabla 3.

Tabla 3: Comunicación con el cliente o usuario. Tabla de contingencia

\begin{tabular}{|c|c|c|c|c|c|c|c|c|c|c|c|c|c|c|c|c|c|}
\hline & & & & & omun & hicaci & ión ce & on el & clien & te 0 & usua & ario & & & & & \\
\hline & & & & & & ctivid & $\begin{array}{r}\text { dades } \\
\text { co }\end{array}$ & $\begin{array}{l}\text { para } \\
\text { on el }\end{array}$ & $\begin{array}{l}\text { esta } \\
\text { client }\end{array}$ & $\begin{array}{l}\text { blece } \\
\text { te o u }\end{array}$ & $\begin{array}{l}\text { er col } \\
\text { usuar }\end{array}$ & muni & icació & & & & Total \\
\hline & & & 2 & 3 & 3 & 3 & 4 & 4 & 4 & 4 & 5 & 5 & 5 & 5 & 5 & 6 & \\
\hline & & Recuento & 1 & 0 & 1 & 0 & 1 & 0 & 2 & 0 & 0 & 1 & 0 & 0 & 0 & 0 & 6 \\
\hline $\begin{array}{l}0 \\
0 \\
0 \\
0 \\
0 \\
0\end{array}$ & 1 & $\begin{array}{l}\text { Frecuencia } \\
\text { esperada }\end{array}$ & .2 & .5 & 1.0 & .2 & .2 & .5 & 1.4 & .5 & .2 & .2 & .2 & .2 & .2 & .2 & 6.0 \\
\hline 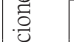 & & Recuento & 0 & 2 & 3 & 0 & 0 & 2 & 0 & 0 & 0 & 0 & 0 & 0 & 0 & 1 & 8 \\
\hline 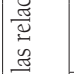 & 2 & $\begin{array}{l}\text { Frecuencia } \\
\text { esperada }\end{array}$ & .3 & .6 & 1.3 & .3 & .3 & .6 & 1.9 & .6 & .3 & .3 & .3 & .3 & .3 & .3 & 8.0 \\
\hline$\because$ & & Recuento & 0 & 0 & 0 & 1 & 0 & 0 & 0 & 0 & 0 & 0 & 0 & 0 & 1 & 0 & 2 \\
\hline 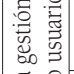 & 3 & $\begin{array}{l}\text { Frecuencia } \\
\text { esperada }\end{array}$ & .1 & .2 & .3 & .1 & .1 & .2 & .5 & .2 & .1 & .1 & .1 & .1 & .1 & .1 & 2.0 \\
\hline $\begin{array}{ll}\pi & 0 \\
\widetilde{\pi} & \Xi\end{array}$ & & Recuento & 0 & 0 & 0 & 0 & 0 & 0 & 1 & 0 & 1 & 0 & 0 & 0 & 0 & 0 & 2 \\
\hline 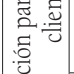 & 3 & $\begin{array}{l}\text { Frecuencia } \\
\text { esperada }\end{array}$ & .1 & .2 & .3 & .1 & .1 & .2 & .5 & .2 & .1 & .1 & .1 & .1 & .1 & .1 & 2.0 \\
\hline 胥 & & Recuento & 0 & 0 & 0 & 0 & 0 & 0 & 0 & 0 & 0 & 0 & 1 & 0 & 0 & 0 & 1 \\
\hline 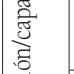 & 4 & \begin{tabular}{|l|} 
Frecuencia \\
esperada
\end{tabular} & .0 & .1 & .2 & .0 & .0 & .1 & .2 & .1 & .0 & .0 & .0 & .0 & .0 & .0 & 1.0 \\
\hline 芯 & & Recuento & 0 & 0 & 0 & 0 & 0 & 0 & 3 & 2 & 0 & 0 & 0 & 1 & 0 & 0 & 6 \\
\hline $\begin{array}{l}\text { E్ } \\
\text { 号 }\end{array}$ & 4 & $\begin{array}{l}\text { Frecuencia } \\
\text { esperada }\end{array}$ & .2 & .5 & 1.0 & .2 & .2 & .5 & 1.4 & .5 & .2 & .2 & .2 & .2 & .2 & .2 & 6.0 \\
\hline & & Recuento & 1 & 2 & 4 & 1 & 1 & 2 & 6 & 2 & 1 & 1 & 1 & 1 & 1 & 1 & 25 \\
\hline Total & & $\begin{array}{l}\text { Frecuencia } \\
\text { esperada }\end{array}$ & 1.0 & 2.0 & 4.0 & 1.0 & 1.0 & 2.0 & 6.0 & 2.0 & 1.0 & 1.0 & 1.0 & 1.0 & 1.0 & 1.0 & 25.0 \\
\hline
\end{tabular}




\begin{tabular}{|l|c|c|c|}
\hline \multicolumn{4}{|c|}{ Pruebas de Ji-cuadrada } \\
\hline & Valor & gl & Sig. asintótica (bilateral) \\
\hline Ji-cuadrada de Pearson & $97.309^{*}$ & 65 & .006 \\
\hline Razón de verosimilitudes & 62.490 & 65 & .565 \\
\hline Asociación lineal por lineal & 3.959 & 1 & .047 \\
\hline $\mathrm{N}^{\circ}$ de casos válidos & 25 & & \\
\hline${ }^{*} 84$ casillas (100.0\%) tienen una frecuencia esperada inferior a 5. La frecuencia mínima esperada es .04.
\end{tabular}

Fuente: Elaboración propia.

\section{Conclusiones}

En la diversidad reside la riqueza potencial para adaptarse a un mundo globalizado que cambia constantemente. Debido a esto las instituciones, organizaciones o empresas pueden aparecer en un gran número de formas. En el ámbito de la educación superior los cambios en las políticas educativas inciden directamente tanto en la gestión y los procesos de enseñanza-aprendizaje dentro y fuera del aula como en la estructura de contenidos y en las estrategias a desarrollar. Es así como continuamente los investigadores, docentes y administrativos procuran implementar procesos, técnicas educativas y herramientas que requieran de una metodología clara y precisa para establecer las bases que propiciarán un cambio que no se produzca al azar, sino como resultado de una construcción científica válida para beneficio propio y de la comunidad.

La implementación y certificación de sistemas de gestión de la calidad bajo la Norma ISO 9001 constituye un gran reto, ya que implica la adopción de una nueva filosofía y una profunda transformación en la cultura laboral, de modo que se requiere de una intervención radical en la cultura social para el logro de nuevas formas de hacer las cosas que satisfagan las necesidades de los usuarios y conseguir ese valioso producto de la nueva economía: el conocimiento. Los hallazgos de la investigación en relación con la implementación de sistemas de gestión de la calidad bajo la Norma ISO 9001 en las instituciones públicas de educación superior permiten manifestar lo siguiente:

- Se puede contar con un sistema de gestión de la calidad certificado sin la existencia de una cultura de la calidad en la institución. Al no encontrarse una correlación significativa entre las actividades para el desarrollo de una cultura de la calidad y la implementación 
de una estructura administrativa para la gestión de la misma, se observa que es necesario hacer énfasis o reconsiderar las acciones para el logro de un liderazgo comprometido con la gestión de la calidad. Es de vital importancia que los directivos estén completamente comprometidos y participen activamente en el proceso del cambio organizacional que significa el desarrollo de una cultura de la calidad.

- En una institución pública de educación superior los procesos clave se corresponden con las funciones sustantivas de la institución. En los sistemas de gestión de la calidad se detecta una falta de procesos relativos a las actividades académicas. Pese a que existen otras formas aceptadas de valorar la calidad de estas actividades mediante los procesos de acreditación de programas educativos, los distintos índices de impacto científico de la investigación y la capacidad de transferencia de tecnología a los sectores productivos y sociales, al no encontrarse una correlación significativa entre la definición de procesos del sistema de gestión de la calidad y el proceso de implementación e indicadores del sistema de gestión de la calidad en los sistemas, es evidente que no se aplica un enfoque basado en procesos y, por ende, no se propicia la mejora continua en este aspecto. Por ello es necesario reconsiderar o reorientar la forma de la aplicación del enfoque basado en procesos: lo que se puede medir, se puede controlar; así como también lo que se puede controlar, se puede mejorar.

- Conocer las percepciones, actitudes y expectativas de los clientes o usuarios en relación con las actividades de una institución como las del estudio proporciona más oportunidades para tomar mejores decisiones. Al encontrarse una correlación significativa entre las actividades para establecer comunicación con el cliente o usuario y la formación/capacitación para la gestión de las relaciones con estos se detecta la aplicación de un enfoque orientado a ellos, estableciéndose una comunicación para beneficio mutuo.

De acuerdo con los lineamientos del Programa Integral de Fortalecimiento Institucional para el logro de mejores niveles de calidad en los programas educativos y servicios que ofrecen las instituciones públicas de educación, se implementan, certifican y se da seguimiento a los sistemas de gestión de la calidad bajo la Norma 
ISO 9001, con el fin de mejorar la calidad en la educación superior. Sin embargo, los resultados del estudio muestran evidencia de que estos sistemas se utilizan para cumplir con el requisito de una política pública, pero no como una herramienta valiosa para la mejora de la calidad en la educación superior. Si bien se encuentran diversos procesos administrativos dentro de los alcances de estos sistemas de gestión, la casi total ausencia de procesos académicos como los de acreditación de programas educativos por parte del Consejo Para la Acreditación de la Educación Superior y su aplicación, la mejora de la calidad y permanencia de los programas educativos de posgrado que lograron su ingreso al Programa Nacional Posgrados de Calidad, entre otros, muestra que las universidades públicas mexicanas desaprovechan las ventajas y beneficios que ofrece una certificación bajo la Norma ISO 9001: 2008.

Al fomentar la participación de las instituciones públicas en el marco del proceso de formulación y actualización periódica del Programa Integral de Fortalecimiento Institucional se ha buscado, en el ámbito de la autonomía de las universidades, generar valor agregado al desarrollo de las instituciones y construir un esquema de aprendizaje colectivo, por medio de la cultura de la planeación participativa y la evaluación externa, teniendo como beneficio principal la mejora de sus propuestas institucionales y, por ende, la mejora en los resultados de sus indicadores. Es de esta forma que se contribuye a la mejora continua de la calidad de la educación superior, por lo que es estratégico continuar con la implementación de sistemas de gestión de la calidad en este tipo de universidad, así como también apoyar el fortalecimiento del programa para el fortalecimiento institucional señalado e incrementar los recursos que se asignan en el marco del programa para beneficio mutuo.

\section{Comentarios finales}

El conocimiento asociado a un individuo o una entidad y a una serie de capacidades organizativas se transforma en capital intelectual, es decir, en ese valor intangible integrado por el conocimiento, la experiencia aplicada, la habilidad profesional, la estrategia organizacional y las relaciones con el usuario o el cliente, todos ellos elementos que a 
una organización o institución le dan una ventaja competitiva en el mercado.

Por otra parte, la misma dinámica de la sociedad del conocimiento requerirá de nuevas formas de aprender, nuevas competencias y habilidades y, por lo tanto, en todos los niveles de la educación se deberá contemplar una apertura a un mundo globalizado en constante cambio.

Será necesario también reconsiderar el papel que juegan tanto los docentes como los estudiantes, junto con la aplicación de las tecnologías de la información, comunicación y colaboración, ya que todo ello redundará en una mejora en la docencia y en la investigación.

En el contexto de la rápida trasformación que ocasiona la globalización, el reto es no quedarse atrás y evitar la inercia, ya que el conocimiento considerado como un producto obliga a las universidades a revisar su misión y sus objetivos, redefinir sus metas y restablecer su visión; de no hacerlo, se perciben amenazas respecto de su estatus y quizás se pone en riesgo su existencia. Lo anterior no implica que las universidades se conviertan en organizaciones empresariales, sino que más bien invita a considerar un nuevo enfoque que responda a los nuevos planteamientos que la sociedad globalizada impone, los que no deben ser ignorados.

\section{Referencias bibliográficas}

Abreu, J.P., (2013) «Las conceptualizaciones sobre la globalización a escala planetaria (I)». [En línea]. Caracas, Venezuela disponible en http:// www.aporrea.org/ideologia/a74271.html [Recuperado el 29 de enero de 2013]

Arcos, J., Hernández, G. y V. Alcántar, (2009) La certificación de los sistemas de gestión de la calidad con la Norma ISO 9001:2000 en la universidad pública. México, Universidad Autónoma de Baja California.

Aréchiga, H. y R. De Thierry, (2003) Antecedentes, situación actual y perspectivas de la evaluación y acreditación de la educación superior en México, documento para IESALC-UNESCO. México, Consejo para la Acreditación de la Educación Superior. 
Cisneros, R., (2003) Más allá de las normas ¿Por qué certificarse en ISO/QS-9000 o ISO/TS-16949 no es suficiente? México, Panorama Editorial, S.A. de C.V.

Clark, B.R., (2000) Creando universidades innovadoras: estrategias organizacionales para la transformación. México, Coordinación de Humanidades UNAM.

Cohen, M.D.; March, J.G. y J.P. Olsen, (1972) «A Garbage Can Model of Organizational Choice» en Administrative Science Quarterly. Vol. 17, N ${ }^{\circ}$ 1. Marzo 1972, pp. 1-25. Disponible en http://www.unc.edu/ fbaum/ teaching/articles/Cohen_March_Olsen_1972.pdf

Crosby, P.B., (1987) La calidad no cuesta: el arte de asegurar la calidad. México, Compañía Editorial Continental, S.A. de C.V.

De la Orden, A.; Asensio, I.; Carballo, R.; Fernández, M.J.; Fuentes, A.; García, J.M.; Guardia, S. y M. Navarro, (1997) «Desarrollo y validación de un modelo de calidad universitaria como base para su evaluación» en Relieve [En línea].Vol. 3, No 1-2. Enero-Junio, Universidad Complutense de Madrid, disponible en: http://www.uv.es/RELIEVE/ v3n1/RELIEVEv3n1_2.htm [Recuperado el 5 de marzo de 2007].

Del Castillo, G., (2006) «Una propuesta analítica para el estudio del cambio en las instituciones de educación superior», en Revista Perfiles Educativos, Vol. 28, No 111. Enero-Marzo, pp. 52-53.

Deming, W.E., (1986) Out of the Crisis: Quality, Productivity and Competitive Position. Estados Unidos de América, Cambridge University Press.

Esponda, A.; Palavicini, J. y G. Navarrete, (2001) Hacia una calidad más robusta con ISO 9000: 2000. México, Panorama Editorial.

FECIES, (2012) Libro de resúmenes de los trabajos aceptados en el IX Foro Internacional sobre la Evaluación de la Calidad de la Investigación y de la Educación Superior. Ramírez, M.T.; Bermúdez, M.P. e I. Teva (Comps.). [En línea]. España, disponible en http://www.ugr.es/ aepc/ IXFORO/LIBRORESUMENESIXFORO.pdf [Recuperado el 12 de junio de 2013]

Ferlie, E.; Pettigrew, A.; Ashburner, L. y L. Fitzgerald, (1996) The New Public Management in Action. Reino Unido, Oxford University Press.

Fusarelli, L.D. y B. Johnson, (2004) «Educational Governance and the New Public Management», en Public Administration and Management: An Interactive Journal. Vol. 9, № 2. Abril-Junio, pp. 118-127.

Gobierno de los Estados Unidos Mexicanos, (2007) Plan Nacional de Desarrollo 2007-2012, Presidencia de la República, Lic. Felipe Calderón Hinojosa. México, Autor. 
Gruening, G., (2001) «Origin and theoretical basis of New Public Management», en International Public Management Journal. $N^{\circ} 4$. Spring, pp.1-25.

Hernández, R.; Fernández, C. y P. Baptista, (2006) Metodología de la Investigación. Cuarta edición. México, McGraw-Hill.

Hood, C., (1991) «A Public Management for All Seasons?», en Public Administration. Vol. 69, Issue 1, Marzo, pp. 3-19.

Ibarra, E., (2003) «Efectos institucionales de las políticas de modernización universitaria en México: autonomía, gobernabilidad y nuevas formas de organización», en Revista de Investigación Educativa, Estudios del Desarrollo [En línea]. No 1, Universidad Mayor de San Simón, Cochabamba, Bolivia disponible en http://estudiosdeldesarrollo.net/ coleccion_america_latina/hacia_una_politica_de_edo/Hacia8.pdf [Recuperado el 31 de octubre de 2013]

Ibarra, E., (2009) «Exigencias de organización y gestión de las universidades públicas mexicanas: de su pasado político a sus mercados presentes», en Cazés, D.; Ibarra, E. y L. Porter (Coord.), Las universidades públicas mexicanas en el año 2030: examinando presentes, imaginando futuros. México, CEIICH-UNAM/UAM-Cuajimalpa.

International Organization for Standardization - ISO, (2005) Norma Internacional. Traducción oficial, Sistemas de gestión de la calidad Fundamentos y vocabulario, Número de referencia ISO 9000:2005. Suiza, Secretaría Central de ISO.

International Organization for Standardization - ISO (2008) Norma Internacional. Traducción oficial, Sistemas de gestión de la calidad Requisitos, Número de referencia ISO 9001:2008. Suiza, Secretaría Central de ISO.

Ishikawa, K., (1988) ¿Qué es el control total de la calidad? La modalidad japonesa. México, Editorial Norma S. A.

Juran, J.M. y F.M. Gryna, (1993) Manual de Control de Calidad. Cuarta edición. España, McGraw-Hill/Interamericana.

Kasperaviciute, R., (2013) «Application of ISO 9001 and EFQM Excellence Model within Higher Education Institutions: Practical Experiences Analysis», en [En línea]. No 1, Annual Journal 2013, Social Transformations in Contemporary Society, disponible en http://stics. mruni.eu/wp-content/uploads/2013/06/81-92.pdf [Recuperado el 1 de noviembre de 2013]

Méndez, J.C.; Jaramillo, D. e I. Serrano, (2006) Gestión de la calidad en procesos de servicios y productivos. México, Instituto Politécnico Nacional. 
Mora, J.G., (2004) «La necesidad del cambio educativo para la sociedad del conocimiento», en Revista Iberoamericana de Educación. N ${ }^{\circ} 35$. MayoAgosto, pp. 13-37.

Organización para la Cooperación y el Desarrollo Económicos (2012). Education at a Glance 2012: OECD Indicators. OCDE Publishing. Doi: 10.1787/eag-2012-en

Pérez, C., (1999). Control estadístico de la calidad, teoría, práctica y aplicaciones informáticas. México, Alfaomega Grupo Editor, S.A. de C.V.

Reimers, F. y E. Villegas-Reimers, (2005) «Sobre la calidad de la educación y su sentido democrático», en Revista PRELAC, Educación para Todos. [En línea] Vol. 2, Julio 2005, Banco Interamericano de Desarrollo, disponible en http://www.oei.es/valores2/prelac_2_reimers.pdf [Recuperado el 25 de julio de 2007]

Rubio, J., (Coord.) (2006) La politica educativa y la educación superior en México. 1995-2006: Un balance. México, Secretaría de Educación Pública y Fondo de Cultura Económica.

Salmi, J., (2009) The Challenge of Establishing World-Class Universities. Estados Unidos de América, The International Bank for Reconstruction and Development/The World Bank.

Scott, A., (2013) New Public Management in Perspective: Higher Education Reform in the UK and Austria. Austria, Department of Sociology, University of Innsbruck.

Secretaria de Educación Pública, (2005) Programa Integral de Fortalecimiento Institucional 2001-2006 de La ENBA, Versión 3.1, Proyecto: Sistema de Gestión de la Calidad. México.

Secretaria de Educación Pública, (2012) Programa Integral de Fortalecimiento Institucional: por la mejora y el aseguramiento de la calidad de la educación superior. Guía para formular el Programa Integral de Fortalecimiento Institucional 2012-2013. Disponible en http://pifi.sep.gob.mx/ScPIFI/ GPIFI_2012/pdfs/Guia_PIFI_2012-2013.pdf

Secretaria de Educación Pública, (2013) La estructura del sistema educativo mexicano. Dirección General de Acreditación, Incorporación y Revalidación. México. Disponible en http://www.sep.gob.mx/work/ models/sep1/Resource/1447/1/images/sistemaedumex09_01.pdf

Subsecretaría de Educación Superior, (2012) Educación superior pública, universidades públicas estatales. México, Secretaría de Educación Pública. Disponible en http://www.ses.sep.gob.mx/wb/ses/universidades_ publicas_estatales 
Senlle, A. y N. Gutiérrez, (2005) Calidad en los servicios educativos. España. Ediciones Díaz de Santos.

SPSS Inc., (2006) SPSS 15.0 Brief Guide. Estados Unidos de América.

Unesco, (2005) Hacia las sociedades del conocimiento. Francia, Ediciones Unesco.

Universidad Autónoma de Baja California (2007). Informe de Rectoría 2007. México. Disponible en http://www.uabc.mx/planeacion/informe/ informe2007/informe.pdf 